\title{
Utilización en el aula de herramientas de gamificación para incrementar la motivación del alumnado por la asignatura Dirección de Operaciones del Grado en Administración y Dirección de Empresas Use of gamification tools in the classroom to increase student motivation for the subject Operations Management of the Degree in Business Administration and Management
}

\author{
Miguel Ángel Montañés-Del Río ${ }^{1}$, Vanessa Rodríguez Cornejo ${ }^{1}$, Margarita Ruiz Rodríguez ${ }^{1}$, Jaime Sánchez Ortiz ${ }^{1}$ \\ miguelangel.montanes@uca.es, vanesa.rodriguez@uca.es,margarita.ruiz@uca.es, jaime.sanchez@uca.es \\ ${ }^{1}$ Departamento de Organización de Empresas \\ Universidad de Cádiz \\ Cádiz, España
}

\begin{abstract}
Resumen- Para la mayoría del alumnado universitario las lecciones magistrales impartidas en el aula son tediosas, generándole una desmotivación traducida en su pasiva actitud. La distracción de los dispositivos móviles de comunicación que portan, cuando no los utilizan con fines educativos, empeora el problema. El resultado es su falta de compromiso con su aprendizaje, demostrado con la caída de asistentes a clase, y con la obtención de resultados académicos mediocres. Una posible solución es incorporar a las clases actividades lúdicas que, implementadas con las nuevas tecnologías ya presentes en la vida diaria, trasladen al ámbito educativo las bondades del juego. Durante el curso 2018/2019, y para mejorar la motivación y las calificaciones del alumnado de Dirección de Operaciones II, asignatura del cuarto curso del Grado en Administración y Dirección de Empresas de la Universidad de Cádiz, se gamificaron las clases impartidas en los Campus de Jerez de la Frontera y de Algeciras utilizando la app gratuita Kahoot! y los teléfonos móviles del alumnado. Aun existiendo otros muchos factores explicativos, la experiencia supuso notables mejoras respecto al curso 2017/2018, aumentando la motivación (el alumnado no presentado a examen descendió un $21,7 \%$ ) y mejorando las calificaciones (la media de aprobados creció un 7,41\%).
\end{abstract}

Palabras clave: Gamificación, Kahoot!, Dirección de Operaciones, Motivación

Abstract- For the majority of university students the master classes given in the classroom are tedious, generating a lack of motivation translated into their passive attitude. The distraction of the mobile communication devices they carry, when not used for educational purposes, worsens the problem. The result is their lack of commitment to their learning, demonstrated by the fall in class attendance, and the achievement of mediocre academic results. A possible solution is to incorporate to the classes playful activities that, implemented with the new technologies already present in daily life, transfer to the educational environment the benefits of the game. During the academic year 2018/2019, and in order to improve the motivation and the grades of the students of Operations Management II, subject of the fourth year of the Degree in Business Administration and Management of the University of Cadiz, the classes given in the Campus of Jerez de la Frontera and Algeciras were gamified using the free app Kahoot! and the mobile phones of the students. Even though there were many other explanatory factors, the experience meant notable improvements with respect to the academic year 2017/2018, increasing motivation (the number of students not presented to the exam fell by $21.7 \%$ ) and improving grades (the average number of passing grades grew by $7.41 \%$ ).

Keywords: Gamification, Kahoot!, Operations Management, Motivation

\section{INTRODUCCIÓN}

El proceso educativo basado en las lecciones magistrales se ha visto influido por el rápido desarrollo de las nuevas tecnologías, modificándose los roles desempeñados por docentes y discentes (Aleksić-Maslać, Rašić, \& Vranešić, 2018). En educación superior la pasiva respuesta del alumnado muestra la ineficiencia de este método (Larsen, 2006), que llega a ser tedioso para las nuevas generaciones, y convierte en un reto mantener al alumnado atento en clase durante toda la jornada lectiva (Aleksić-Maslać et al., 2018).

Así, el actual panorama educativo requiere incorporar los avances tecnológicos para permitir mejorar la calidad de la enseñanza y del proceso de aprendizaje (Molina Álvarez, Ortiz Colón, \& Agreda Montoro, 2017). Frente a los tradicionales métodos de aprendizaje que han demostrado falta de motivación en el alumnado (Simões, Redondo, \& Vilas, 2013), irrumpen los juegos como uno de los medios para poder motivar y atraer la atención de estas nuevas generaciones de estudiantes a través, entre otros factores, de la competición (Hanus \& Fox, 2015). Para Kenny y McDaniel (2011), los juegos fomentan la motivación del alumnado gracias a su componente lúdico, confirmándose la relación motivación-gamificación (Molina Álvarez et al., 2017).

Los juegos representan, por lo tanto, una gran oportunidad para evitar el abandono del alumnado, la desmotivación, la desgana, o la ausencia de compromiso en su proceso de enseñanza-aprendizaje (Mérida Serrano, Angulo Romero, 
Jurado Bello, \& Diz Pérez, 2011), por lo que su incorporación en la educación persigue fomentar la motivación, el compromiso y determinados comportamientos (Lee \& Hammer, 2011).

Por otra parte, el creciente uso diario de recursos tecnológicos ha conducido a que terminen por implantarse también en las aulas, respondiendo así de forma efectiva a las demandas del alumnado (Curto Prieto, Orcos Palma, Blázquez Tobías, \& Molina León, 2019). En este sentido, la utilización de sistemas de respuesta personal (Student Response Systems) en las clases puede no solo estimular una mayor asistencia debido a su popularidad entre el alumnado (Castilla, Romana, \& López-Terradas, 2013), sino también aumentar el disfrute por un curso docente (Abrahamson, 1998).

La utilización en clase de la aplicación gratuita Kahoot! trata de aumentar la motivación del alumnado $\mathrm{y}$, en consecuencia, mejorar sus calificaciones (San-Miguel, Megías, \& Serna, 2017), por lo que comprobar si las calificaciones del alumnado en la asignatura Dirección de Operaciones II, impartida en el Grado en Administración y Dirección de Empresas de la Universidad de Cádiz durante el curso académico 2018-2019, han mejorado respecto a las del curso anterior a consecuencia de la mayor motivación de aquel por el uso en clase de cuestionarios realizados con Kahoot!, es el principal objetivo de este artículo.

\section{CONTEXTO}

\section{A. Gamificación}

Las organizaciones, Universidades inclusive, se enfrentan a una crisis de compromiso debido al limitado tiempo del que disponen las personas que las integran. Para evitar perderlo, estas últimas crean escudos que tan solo unas pocas organizaciones atraviesan al generar cierta vinculación con ellas. En este sentido, la gamificación es una estrategia emergente (Burke, 2014).

La definición de gamificación es compleja y poco consensuada (Molina Álvarez et al., 2017). De origen anglosajón, el término describe la utilización de técnicas, elementos y dinámicas que, propias de los juegos (Alsawaier (2018) señala principalmente a los video juegos), se trasladan a otros ámbitos ajenos a los mismos (Jaber et al., 2016) para intentar transmitir unos contenidos que, por medio de una experiencia lúdica, potencien aspectos como la motivación, la implicación, el aprendizaje o la diversión (Molina Álvarez et al., 2017), pero también la concentración y el esfuerzo entre grupos de personas (Castilla et al., 2013).

Pero el uso de dinámicas y mecánicas de juego en entornos no lúdicos no es algo nuevo (Molina Álvarez et al., 2017). Desde hace tiempo se "acumulan millas" al volar con las aerolíneas lo que, unido a la aparición de dispositivos móviles $\mathrm{y}$ aplicaciones descargables, ha servido para popularizar su utilización en otros ámbitos (Smith-Robbins, 2011). En consecuencia, se haría hincapié sobre dinámicas de juegos digitales tales como avatares, puntos, niveles, recompensas virtuales, entre otras muchas (Alsawaier, 2018).

El esfuerzo por acumular puntos y escalar categorías tras completar una serie de pruebas es extrapolable al sistema educativo, donde la titulación otorgada por una Institución acreditada sería no solo la categoría alcanzada sino también una muestra de distinción, y las calificaciones logradas en cada asignatura, las puntuaciones (Smith-Robbins, 2011).

Es mucho el alumnado que percibe la educación tradicional como algo aburrido y en ocasiones poco eficaz, por lo que el profesorado busca continuadamente nuevos métodos de enseñanza para motivarlo y captar su compromiso con las asignaturas (Contreras Espinosa \& Eguia, 2016). La motivación del alumnado en el aula constituye, por lo tanto, una de las mayores preocupaciones del docente (Castilla et al., 2013) porque, cuando se está motivado, se obtienen mejores resultados (Santana Cabello \& García Juan, 2018). La gamificación incorpora estrategias para hacer clases más motivadoras y que el alumnado quiera aprender (de Soto García, 2018). Se aprende mejor cuando se está motivado (Bergin \& Reilly, 2005), y cuando el alumnado se divierte al realizar una tarea, la información que recibe se fija en su cerebro generando mejores aprendizajes (Molina Álvarez et al., 2017).

Pero la gamificación no consiste en un juego en sí mismo. Antes bien, implica utilizar los elementos básicos que lo componen en contextos o entornos que no son lúdicos, como en la enseñanza, introduciendo en el aula recompensas, insignias, puntuaciones y dinámicas competitivas (Simões et al., 2013). Las principales definiciones de gamificación, de hecho, subrayan que no se trata tanto de crear un juego como de incorporar en otras actividades aquello que lo hace tan atractivo, siendo por ello que la teoría de la gamificación supone que el diseño apropiado de un juego puede cambiar el comportamiento humano y aumentar su productividad (Jakubowski, 2014).

En consecuencia, la gamificación es otra actividad de aprendizaje adicional cuyos fines pedagógicos trascienden el objetivo de la motivación (Molina Álvarez et al., 2017). Por lo tanto, los juegos adaptados al ámbito educativo deberían contar con dinámicas centradas en retos, recompensas, etc., que hicieran las clases más atractivas para el alumnado y que, además, pudiesen mejorar sus resultados académicos al fomentar una participación más activa en clase (de Soto García, 2018).

Para Díaz Cruzado y Troyano Rodríguez (2013), dos son los grupos en que se suelen dividir las actividades gamificadas: (1) juegos educativos convencionales que no precisan de un soporte electrónico, y; (2) actividades con soporte digital que, a diferencia de los anteriores, permiten incluir imágenes, sonidos, vídeos, etc., y que conforman un espacio de juego más atractivo, siendo Kahoot! y Socrative las aplicaciones de este tipo más usadas en las aulas.

\section{B. Ventajas e inconvenientes de la gamificación}

Algunos beneficios derivados de utilizar actividades gamificadas en el aula son (Kay \& LeSage, 2009): (1) que al generar mayor interacción entre iguales para discutir posibles soluciones y elegir la más apropiada, se mejora el proceso de enseñanza-aprendizaje; (2) que al aumentar la atención del alumnado, más concentrado en lo que debe hacer en clase, y participando de forma activa al competir con otros compañeros, se mejora el ambiente, y; (3) que al obtenerse una retroalimentación instantánea sobre los resultados, mejora el sistema de evaluación del alumnado.

Las opiniones contrarias a la gamificación se basan, por un lado, en la idea de que la simple suma de puntos no hará que 
las personas quieran, de forma automática, obtenerlos. Y, por otro lado, en el hecho de que permitir que una persona pueda conseguir más puntos que otra no ha de servirle, necesariamente, de motivación (Smith-Robbins, 2011). La gamificación, por otra parte, puede hacer ver al alumnado que solo debería aprender cuando se le facilitaran recompensas externas (Lee \& Hammer, 2011).

\section{Kahoot! como Game-based Student Response System}

Kahoot!, como integrante de los Sistemas de Respuesta del Alumnado Basados en el Juego (GSRS por su acrónimo inglés), es una popular y gratuita herramienta de eLearning, fácil de aprender y de usar, que consigue la participación y compromiso del alumnado, añadiendo vitalidad y apoyos meta-cognitivos (retroalimentación instantánea, p.e.) a las clases de educación superior con muy poco esfuerzo del profesorado (Plump \& LaRosa, 2017). El docente lanza desde su ordenador el juego, que se proyecta sobre una pantalla gigante, y su alumnado se conecta mediante un dispositivo móvil a una página web (https://kahoot.it/) desde la que introduce un código (actualmente, un PIN de seis dígitos) que le permitirá participar en el cuestionario (Parra Santos, Molina Jordá, \& Casanova Pastor, 2018). Al no ser preciso tener que registrarse, ni facilitar su auténtico nombre, el alumnado puede concursar de forma anónima $\mathrm{y}$, en consecuencia, sentirse menos presionado por los fallos. Al terminar la actividad, Kahoot! facilita automáticamente los resultados obtenidos, por lo que se pueden ver los aciertos y los errores cometidos para establecer un período de debate que, por lo general, suele ser bien recibido por el alumnado al no apreciarlo como una crítica de su trabajo por estar en un ambiente lúdico (de Soto García, 2018).

Se decidió utilizar Kahoot! porque la inclusión de cuestionarios tipo test facilita el aprendizaje y la memoria (Toppino \& Gerbier, 2014), permitiendo comprobar al alumnado lo que sabe y lo que no, y así concentrar sus esfuerzos en el estudio de aquellas áreas con menor conocimiento (Roediger III, Putnam, \& Smith, 2011).

\section{Ventajas e inconvenientes de Kahoot!}

La atención es un elemento de motivación y un prerrequisito para el aprendizaje (Keller, 1987), luego conseguirla del estudiante es una cuestión fundamental para su aprendizaje (Gagne \& Driscoll, 1988), y Kahoot! lo hace al mostrarse en la pantalla distintos colores, y sonar una melodía que recuerda a los videojuegos (de Soto García, 2018). Además de cuestionarios, con Kahoot! se pueden realizar rompecabezas, gráficos y discusiones, lo que reforzaría el aprendizaje (Dellos, 2015).

Tabla 1. Ventajas e inconvenientes al usar Kahoot! en el aula

\begin{tabular}{|l|l|}
\hline \multicolumn{1}{|c|}{ Ventajas } & \multicolumn{1}{c|}{ Inconvenientes } \\
\hline Gratuito & $\begin{array}{l}\text { Tiempo requerido para su } \\
\text { preparación y puesta en práctica }\end{array}$ \\
\hline Fácil de aprender para el profesorado & $\begin{array}{l}\text { Necesidad de conexión } \\
\text { permanente a Internet }\end{array}$ \\
\hline $\begin{array}{l}\text { Procesos simples para el alumnado } \\
\text { (no precisa una cuenta para registrarse } \\
\text {-anonimato- ni descargar aplicación } \\
\text { alguna) }\end{array}$ & $\begin{array}{l}\text { La posibilidad de que el } \\
\text { alumnado copie sus respuestas }\end{array}$ \\
\hline $\begin{array}{l}\text { Compatible con smartphones, tabletas } \\
\text { (dispositivos electrónicos móviles) } \mathrm{u} \\
\text { ordenadores personales }\end{array}$ & $\begin{array}{l}\text { No se pueda marcar más de una } \\
\text { respuesta como correcta }\end{array}$ \\
\hline La obtención de resultados en tiempo & Limitación en el número de \\
\hline
\end{tabular}

\begin{tabular}{|l|l|}
\hline \multicolumn{1}{|c|}{ Ventajas } & \multicolumn{1}{c|}{ Inconvenientes } \\
\hline $\begin{array}{l}\text { real permite al profesorado facilitar } \\
\text { aclaraciones sobre contenidos allí } \\
\text { donde sean precisas }\end{array}$ & $\begin{array}{l}\text { caracteres incluidos en las } \\
\text { respuestas }\end{array}$ \\
\hline $\begin{array}{l}\text { La música, los colores, y la } \\
\text { competitividad, animan e implican al } \\
\text { estudiante }\end{array}$ & $\begin{array}{l}\text { Contar con un porcentaje de clase } \\
\text { desmotivada por haberse } \\
\text { clasificado en las posiciones más } \\
\text { bajas }\end{array}$ \\
\hline
\end{tabular}

Fuente: Adaptado de Plump y LaRosa (2017), Martínez Navarro (2017) y de Soto García (2018)

\section{DESCRIPCIÓN}

\section{A. Información previa de la asignatura a gamificar}

Dirección de Operaciones es impartida en la Universidad de Cádiz (en adelante, UCA) por el Departamento de Organización de Empresas, y se distribuye en dos cursos sucesivos del Grado en Administración y Dirección de Empresas (en lo sucesivo, ADE) donde se muestran, respectivamente, los aspectos estratégicos (Dirección de Operaciones I) y los operativos (Dirección de Operaciones II).

La investigación aquí realizada se refiere a Dirección de Operaciones II (en lo sucesivo, DOPII), con una carga docente de 6 ECTS que se distribuyen entre teoría (4 ECTS) y práctica (2 ECTS), y se imparte con carácter obligatorio en el primer semestre del cuarto curso del plan de estudios del Grado en ADE en tres de los Campus de la UCA: Cádiz, Jerez de la Frontera y Algeciras. La evaluación de esta asignatura considera los siguientes ítems:

- Cuestionarios tipo test de respuesta múltiple y solo una correcta, de carácter voluntario. Para un total de siete de ellos, el peso de este ítem en la nota final es de un $10 \%$.

- Dos ejercicios prácticos individuales, también voluntarios, a realizar en clase y con un peso conjunto igualmente del $10 \%$ en la nota final.

- Examen (obligatorio) presencial. Su peso es el $80 \%$ de la nota final, y abarca dos partes: una teórica y otra práctica. En ambas se debe conseguir un mínimo del 30\% de la nota otorgada a las mismas, también aplicado a cada uno de los tres ejercicios prácticos propuestos. Además, y en su caso, la suma de la nota obtenida en cada una de dichas partes deberá alcanzar el $50 \%$ de la nota otorgada al examen presencial.

La fijación de una nota mínima de corte para aprobar supone, a la vista de la experiencia docente ya acumulada, cierta frustración entre el alumnado. Por ello, se planteó un Proyecto de Innovación y Mejora Docente (en adelante PIMD) basado en la utilización en el aula de herramientas didácticas alternativas como Kahoot!, con el que se pretendió estudiar si era posible motivar al alumnado, y que así obtuviera mejores calificaciones.

\section{B. Diseño de la investigación}

El estudio realizado tomó una muestra de 60 estudiantes de la asignatura DOPII en los Campus de Jerez de la Frontera y de Algeciras durante el curso académico 2018/2019. El Campus de Cádiz no participó en el estudio al haberse reducido drásticamente el alumnado asistente a clase.

Se confeccionaron siete cuestionarios de respuesta múltiple con Kahoot!, uno por cada tema de la programación de DOPII, y en la clase inmediatamente posterior a la terminación de 
cada tema se solicitaba del alumnado asistente la utilización de sus dispositivos móviles para contestarlos.

Al finalizar cada pregunta, tal y como propone Tucker (2012) y ya se ha utilizado en la asignatura Dirección de Operaciones de la Universidad Rey Juan Carlos de Madrid (Díaz-Garrido, Martín-Peña, \& Sánchez-López, 2017), se establecía un breve debate con el alumnado para afianzar los conceptos mostrados en cada una de aquellas y determinar, mediante aprendizaje colaborativo entre los asistentes, el porqué de las respuestas incorrectas.

Además, y en la última de las clases destinadas a la docencia en DOPII, se le entregó al alumnado asistente un cuestionario de opinión final para conocer su grado de motivación con la asignatura, y su nivel de satisfacción con el PIMD. Se había de responder anónima y voluntariamente al objeto de conseguir mayor objetividad, estando su confección basada, previa adaptación al contexto particular de la investigación, en dos trabajos previos: (1) la encuesta de motivación con el curso realizada por Kebritchi, Hirumi y Bai (2010), y; (2) la investigación de San-Miguel, Megías y Serna (2017).

El cuestionario utilizó una escala Likert de 5 puntos sobre unos ítems relacionados con la motivación y la satisfacción (véase la tabla 2). Este tipo de escala suministra una base común para las respuestas a los ítems relacionados con diferentes aspectos de la experiencia universitaria (Douglas, Douglas, \& Barnes, 2006). Otorgar 1 punto significaba estar "Nada de acuerdo", mientras que 2 puntos suponían "Estar de acuerdo" y el valor neutro implicaba conceder 3 puntos. Los valores más altos concedidos en cada ítem, 4 y 5, suponían "Estar muy de acuerdo" y "Estar totalmente de acuerdo", respectivamente.

Tabla 2. Ítems del cuestionario de opinión final en DOPII

\begin{tabular}{|c|c|}
\hline Ítem & Codificación \\
\hline $\begin{array}{l}\text { 1. Conocer la respuesta correcta justo después de } \\
\text { cada pregunta le ha ayudado en su aprendizaje. }\end{array}$ & FDBCK \\
\hline $\begin{array}{l}\text { 2. La realización de este tipo de cuestionarios ayuda } \\
\text { en la asimilación de los contenidos. }\end{array}$ & ASIM_CTDOS \\
\hline $\begin{array}{l}\text { 3. El debate realizado después de cada pregunta del } \\
\text { cuestionario permite entender mejor muchos } \\
\text { conceptos. }\end{array}$ & DEBT \\
\hline $\begin{array}{l}\text { 4. La realización de este tipo de actividad permite } \\
\text { seguir mejor la asignatura. }\end{array}$ & SEG_DOPII \\
\hline $\begin{array}{l}\text { 5. Usted prefiere realizar este tipo de cuestionarios } \\
\text { frente a los que se realizan en pape y son } \\
\text { entregados al profesor para su posterior } \\
\text { evaluación. }\end{array}$ & PRFRCIA_1 \\
\hline $\begin{array}{l}\text { 6. Usted prefiere realizar este tipo de cuestionarios } \\
\text { frente a los que se realizan a través del aula virtual } \\
\text { de la asignatura. }\end{array}$ & PRFRCIA_2 \\
\hline $\begin{array}{l}\text { 7. Le parece un sistema de evaluación continuada } \\
\text { original. }\end{array}$ & EVA_ORIG \\
\hline $\begin{array}{l}\text { 8. Le parece un sistema de evaluación continuada } \\
\text { muy útil. }\end{array}$ & EVA_UTIL \\
\hline $\begin{array}{l}\text { 9. Le gustaría que este tipo de cuestionarios fuera } \\
\text { incluido en otras asignaturas. }\end{array}$ & OT_ASIGN \\
\hline $\begin{array}{l}10 . \quad \text { Los elementos de innovación y mejora } \\
\text { docente aplicados en esta asignatura han } \\
\text { favorecido su comprensión de los contenidos y/o } \\
\text { la adquisición de competencias asociadas a la } \\
\text { misma. }\end{array}$ & COMPR_CPTCIAS \\
\hline
\end{tabular}

\section{Resultados}

En la figura 1 se muestra, para cada ítem representativo de la motivación y de la satisfacción del alumnado en los Campus de Jerez de la Frontera y de Algeciras, la puntuación media otorgada al mismo por el alumnado que cursó DOPII. La media global de dichos ítems fue de 3,718 y la media de la desviación estándar fue de 1,188.

Figura 1. Motivación-satisfacción del alumnado de DOPII

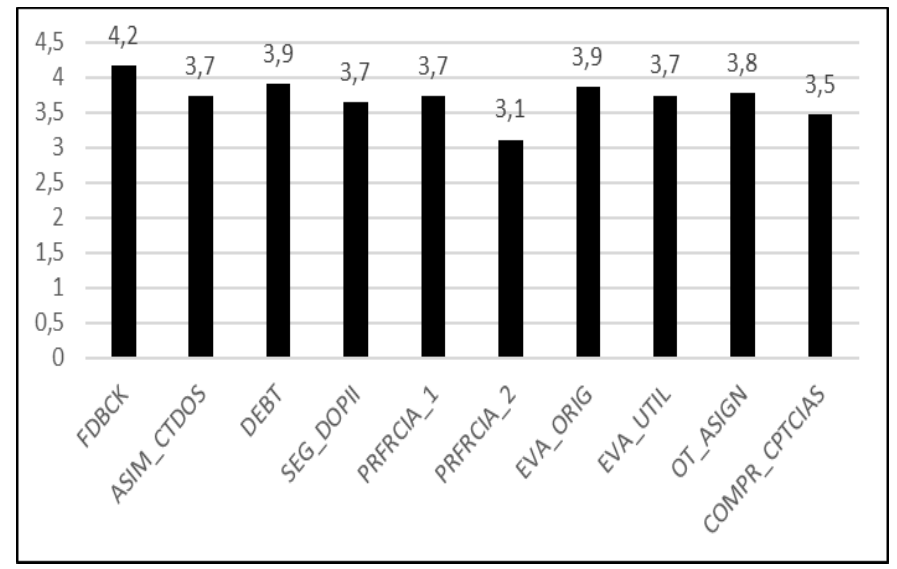

B. Calificaciones en el Campus de Jerez de la Frontera

Tomando como referencia las convocatorias de examen de febrero de 2018 (sin Kahoot!) y de febrero de 2019 (con Kahoot!), el alumnado NO PRESENTADO descendió un $25,64 \%$ (véase la figura 2). Aun la existencia de otros factores explicativos, este dato mostraría una mayor motivación del alumnado por la asignatura tras la introducción de Kahoot! en la docencia, bien por su orientación al resultado, bien por su orientación a la evitación (Alonso Tapia, 2007). La mayor participación aumentó un $18,18 \%$ el alumnado SUSPENSO, pero también incrementó un $24,84 \%$ el alumnado APROBADO, y un $166,67 \%$ el alumnado con NOTABLE, elevándose la nota media de examen un $7,14 \%$.

Figura 2. Calificaciones DOPII del Campus de Jerez de la Frontera

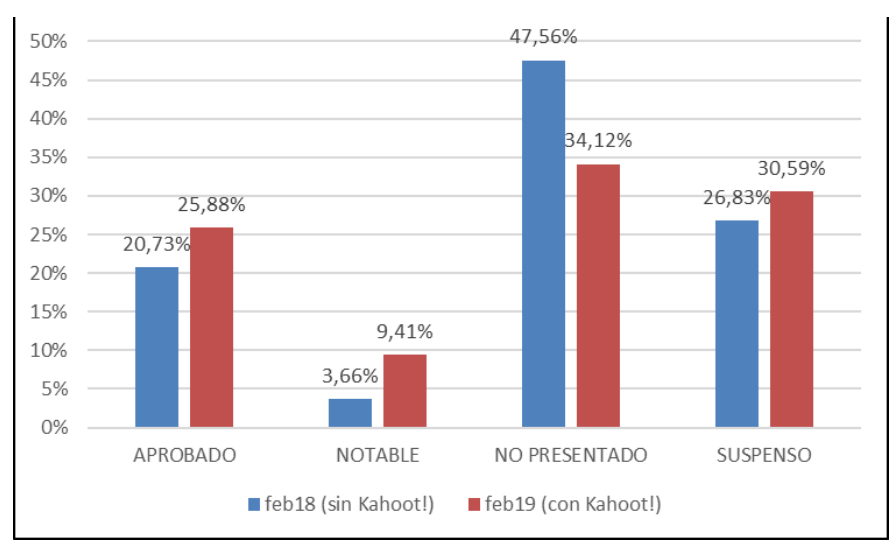

C. Calificaciones en el Campus de Algeciras

En el mismo período de referencia, el alumnado NO PRESENTADO aumentó un 14,29\% mientras el alumnado SUSPENSO descendió un 25,32\%, y el alumnado que superó la asignatura creció un 10,70\% (véase la figura 3).

Figura 3. Calificaciones DOPII del Campus de Algeciras

A. Cuestionario de opinión final 


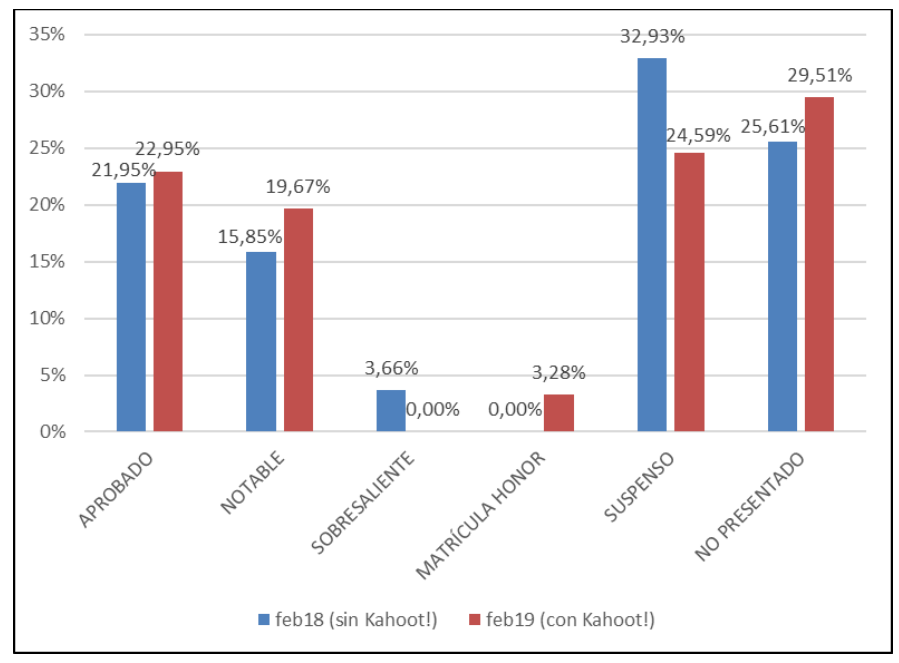

\section{CONCLUSIONES}

El uso de Kahoot! en la asignatura DOPII durante el curso 2018/2019 de la UCA ha mostrado notables mejoras en los rendimientos del alumnado, tanto en porcentaje de aprobados como en incremento de la nota media, lo que estaría en concordancia con las mejoras de resultados académicos obtenidas con procesos de enseñanza-aprendizaje gamificados (Molina Álvarez et al., 2017), y en las mejores calificaciones en exámenes universitarios tras su utilización (Iwamoto, Taitano, Hargis, \& Vuong, 2017).

$\mathrm{Si}$ bien es cierto que dichos resultados han podido deberse a la acción conjunta de otros muchos factores, a la vista del aumento del alumnado que se presentó a examen (respecto del curso inmediato anterior), no lo es menos el hecho de que parece haberse mostrado un aumento de la motivación de aquel. Ello confirmaría que los juegos pueden integrarse en una clase tradicional de educación superior para mejorar el aprendizaje, la motivación y el compromiso (Wang \& Lieberoth, 2016).

\section{REFERENCIAS}

Abrahamson, A. L. (1998). An overview of teaching and learning research with classroom communication systems. Samos International Conference on the Teaching of Mathematics. Samos, Greece.

Aleksić-Maslać, K., Rašić, M., \& Vranešić, P. (2018). Influence of gamification on student motivation in the educational process in courses of different fields. MIPRO 2018: 41st International Convention on Information and Communication Technology, Electronics and Microelectronics. Opatija, Croatia.

Alsawaier, R. (2018). The Effect of Gamification on Motivation and Engagement. International Journal of Information and Learning Technology, 35, 56-79. https://doi.org/10.1108/IJILT-02-2017-0009

Bergin, S., \& Reilly, R. (2005). The influence of motivation and comfort-level on learning to program. In E. A. C. \& S. B. P. Romero, J. Good (Ed.), 17th Workshop of the Psychology of Programming Interest Group.

Burke, B. (2014). Gamify. How Gamification Motivates People to Do Extraordinary Things. https://doi.org/10.4324/9781315230344

Castilla, G., Romana, M. G., \& López-Terradas, B. (2013). Concursando en el aula: la gamificación mediante quizzshow como herramienta de dinamización docente. $X$ Jornadas Internacionales de INnovación Universitaria: Educar Para Transformar. Madrid, España.

Contreras Espinosa, R. S., \& Eguia, J. L. (2016). Gamificación en las aulas universitarias (R. S. Contreras Espinosa \& J. L. Eguia, eds.). Institut de la Comunicació, Universitat Autónoma de Barcelona.

Curto Prieto, M., Orcos Palma, L., Blázquez Tobías, P., \& Molina León, F. J. (2019). Student Assessment of the Use of Kahoot in the Learning Process of Science and Mathematics. Education Sciences, 9(1). Retrieved from https://www.mdpi.com/2227-7102/9/1/55

de Soto García, I. S. (2018). Herramientas de gamificación para el aprendizaje de Ciencias de la Tierra. EDUTEC. Revista Electrónica de Tecnología Educativa, (65), 29 39.

https://doi.org/dx.doi.org/10.21556/edutec.2018.65.1143

Dellos, R. (2015). Kahoot! A digital game resource for learning. International Journal of Instructional Technology and Distance Learning, 12(4), 49-52. Retrieved from www.itdl.org/Journal/apr_15/apr15.pdf

Díaz-Garrido, E., Martín-Peña, M. L., \& Sánchez-López, J. M. (2017). The impact of Flipped Classroom on the motivation and learning of students in Operations Management. Working Papers on Operations Management, $8, \quad 15$. https://doi.org/10.4995/wpom.v8i0.7091

Díaz Cruzado, J., \& Troyano Rodríguez, Y. (2013). El potencial de la gamificación aplicado al ámbito educativo. III Jornadas de Innovación Docente. Innovación Educativa: Respuesta En Tiempos de Incertidumbre. Retrieved from https://idus.us.es/xmlui/handle/11441/59067

Douglas, J., Douglas, A., \& Barnes, B. (2006). Measuring student satisfaction at a UK university. Quality Assurance in Education, 14(3), 251-267. https://doi.org/10.1108/09684880610678568

Gagne, R., \& Driscoll, M. (1988). Essentials of learning for instruction (2nd ed.). Englewood Cliffs, NJ: Prentice Hall.

Hanus, M. D., \& Fox, J. (2015). Assessing the effects of gamification in the classroom: A longitudinal study on intrinsic motivation, social comparison, satisfaction, effort, and academic performance. Computers and Education, $\quad 80, \quad 152-161$. https://doi.org/10.1016/j.compedu.2014.08.019

Iwamoto, D. H., Taitano, E. J., Hargis, J., \& Vuong, K. (2017). Analyzing the Efficacy of the Testing Effect Using Kahoot on Student Performance. Turkish Online Journal of Distance Education, 18(April), 80-93. Retrieved from https://eric.ed.gov/?id=EJ1145220 
Jaber, J. R., Arencibia, A., Carrascosa, C., Ramírez, A. S., Rodríguez-Ponce, E., Melian, C., ... Farray, D. (2016). Empleo de Kahoot como herramienta de gamificación en la docencia universitaria. III Jornadas Iberoamericanas de Innovación Educativa En El Ámbito de Las TIC. Las Palmas de Gran Canaria, España.

Jakubowski, M. (2014). GAMIFICATION IN BUSINESS AND EDUCATION - PROJECT OF GAMIFIED COURSE FOR UNIVERSITY STUDENTS. Developments in Business Simulation and Experiential Learning, 41, 339-342.

Kay, R. H., \& LeSage, A. (2009). Examining the benefits and challenges of using audience response systems: A review of the literature. Computers and Education, 53(3), 819827. https://doi.org/10.1016/j.compedu.2009.05.001

Kebritchi, M., Hirumi, A., \& Bai, H. (2010). The effects of modern mathematics computer games on mathematics achievement and class motivation. Computers and Education, 55(2), 427-443. https://doi.org/10.1016/j.compedu.2010.02.007

Keller, J. M. (1987). Development and use of the ARCS model of instructional design. Journal of Instructional Development, $\quad 10(3), \quad 2-10$. https://doi.org/10.1007/BF02905780

Kenny, R. F., \& McDaniel, R. (2011). The role teachers' expectations and value assessments of video games play in their adopting and integrating them into their classrooms. British Journal of Educational Technology, 42(2), 197-213. https://doi.org/10.1111/j.14678535.2009.01007.x

Larsen, M. D. (2006). Advice for New and Student Lecturers on Probability and Statistics. Journal of Statistics Education, 14(1). Retrieved from $\mathrm{http}: / /$ jse.amstat.org/v14n1/larsen.html

Lee, J., \& Hammer, J. (2011). Gamification in Education: What, How, Why Bother? Academic Exchange Quarterly, 15(2), 1-5.

Martínez Navarro, G. (2017). Tecnologías y nuevas tendencias en educación: aprender jugando. El caso de Kahoot. Opción: Revista de Ciencias Humanas y Sociales, 33(83), 252-277. Retrieved from https://dialnet.unirioja.es/servlet/articulo?codigo $=622833$ 8

Mérida Serrano, R., Angulo Romero, J., Jurado Bello, M., \& Diz Pérez, J. (2011). Student training in transversal competences at the University of Cordoba. European Educational Research Journal, 10(1), 34-52. https://doi.org/10.2304/eerj.2011.10.1.34

Molina Álvarez, J. J., Ortiz Colón, A. M., \& Agreda Montoro, M. (2017). Análisis de la integración de procesos gamificados en Educación Primaria. In Innovación docente y uso de las TIC en educación. Retrieved from http://www.enriquesanchezrivas.es/congresotic/archivos/ Form_Compet_metodos/Ortiz_Otros_2.pdf

Parra Santos, M. T., Molina Jordá, J. M., \& Casanova Pastor,
G. (2018). La Aplicación Kahoot! para Motivar la Participación Activa en el Aula. In Redes de investigación en docencia universitaria. Retrieved from http://rua.ua.es/dspace/handle/10045/85010\#.XJtggMUa94.mendeley

Plump, C. M., \& LaRosa, J. (2017). Using Kahoot! in the Classroom to Create Engagement and Active Learning: A Game-Based Technology Solution for eLearning Novices. Management Teaching Review, 2(2), 151-158. https://doi.org/10.1177/2379298116689783

Roediger III, H. L., Putnam, A. L., \& Smith, M. A. (2011). Ten Benefits of Testing and Their Applications to Educational Practice. Psychology of Learning and Motivation, 55, 1-36. https://doi.org/10.1016/B978-0-12387691-1.00001-6

San-Miguel, T., Megías, J., \& Serna, E. (2017). Gamificación en la universidad II: aprendemos a divertirnos enseñando. Se divierten aprendiendo. IN-RED 2017: III Congreso Nacional de Innovación Educativa y Docencia En Red. https://doi.org/10.4995/inred2017.2017.6837

Santana Cabello, R., \& García Juan, L. (2018). La gamificación en la educación superior. Tecnologías emergentes que motivan al estudio y aumentan el rendimiento. In Las competencias y la sociedad del conocimiento (pp. 155-176). Retrieved from www.memoriascimted.com

Simões, J., Redondo, R. D., \& Vilas, A. F. (2013). A social gamification framework for a K-6 learning platform. Computers in Human Behavior, 29(2), 345-353. https://doi.org/10.1016/j.chb.2012.06.007

Smith-Robbins, S. (2011). This Game Sucks: How to Improve the Gamification of Education. Educause Review, 46, 58-59. Retrieved from https://er.educause.edu/articles/2011/2/this-game-suckshow-to-improve-the-gamification-of-education

Toppino, T. C., \& Gerbier, E. (2014). About practice. Repetition, spacing, and abstraction. Psychology of Learning and Motivation, 60, 113-189. https://doi.org/10.1016/B978-0-12-800090-8.00004-4

Tucker, B. (2012). The Flipped Classroom. Online instruction at home frees class time for learning. Education Next, 12(1), 82-83.

Wang, A. I., \& Lieberoth, A. (2016). The effect of points and audio on concentration, engagement, enjoyment, learning, motivation, and classroom dynamics using Kahoot! In Thomas Connolly \& Liz Boyle (Eds.), 10th European Conference of Game Based Le (p. 9). Retrieved from http://pure.au.dk/portal/en/publications/the-effect-ofpoints-and-audio-on-concentration-engagementenjoyment-learning-motivation-and-classroomdynamics-using-kahoot(f1ddf129-5e63-4101-80dd1050a162c57d).html 